\title{
Integrated Fault Location and Power-Quality Analysis in Electric Power Distribution Systems
}

\author{
A. A. P. Bíscaro, Member, IEEE, R. A. F. Pereira, M. Kezunovic, Fellow, IEEE, and \\ J. R. S. Mantovani, Member, IEEE
}

\begin{abstract}
This paper presents a methodology for automated disturbance analysis and fault location on electric power distribution systems using a combination of modern techniques for network analysis, signal processing, and intelligent systems. New algorithms to detect, classify, and locate power-quality disturbances are developed. The continuous process of detecting these disturbances is accomplished through statistical analysis and multilevel signal analysis in the wavelet domain. The behavioral indices of the current and voltage signals are extracted by employing the discrete wavelet transform, multiresolution analysis, and the concept of signal energy. These indices are used by a number of independent Fuzzy-ARTMAP neural networks, which aim to classify the fault type and the power-quality events. The fault location is performed after the classification process. A real life three-phase distribution system with 134 nodes- $13.8 \mathrm{kV}$ and $7.065 \mathrm{MVA}$ - was used to test the proposed algorithms, providing satisfactory results, attesting that the proposed algorithms are efficient, fast, and, above all, intelligent.
\end{abstract}

Index Terms-Fault location, neural networks, pattern classification, power distribution, power quality (PQ), wavelet transforms.

\section{INTRODUCTION}

$\mathbf{T}$ HE economic and technological growth currently experienced increasingly require higher demand for energy and greater efficiency of the electricity sector. This rapid growth has created good opportunities for research and development of new products and services that meet the new reality of utilities and customers [1]. Currently, energy is viewed as a product and not only as a service and, like every product, becomes quantified by its characteristics, such as quality, price, and accessibility. In modern societies, loads are much more sensitive to power-quality (PQ) events [2], [4], that is, variations and voltage

Manuscript received January 27, 2014; revised June 27, 2014; accepted July 30, 2015. Date of publication August 03, 2015; date of current version March 22,2016 . This work was supported in part by CAPES under Grant 4831-10-1, in part by FAPESP under Grant 13/23590-8, and in part by CNPq under Grant 305371/2012-6. Paper no. TPWRD-00102-2014.

A. A. P. Bíscaro is with Universidade do Estado de Mato Grosso - UNEMAT, Departamento de Engenharia Elétrica, Sinop, MT 78555-000, Brazil (e-mail: aapbiscaro@unemat-net.br).

R. A. F. Pereira and J. R. S. Mantovani are with Faculdade de Engenharia de Ilha Solteira, UNESP - Universidad Estadual Paulista, Departamento de Engenharia Elétrica, Ilha Solteira, SP 15385-000, Brazil (e-mail: ddigo@yahoo.com; mant@dee.feis.unesp.br).

M. Kezunovic is with the Department of Electrical and Computer Engineering, Texas A\&M University, College Station, TX 77843-3128 USA (e-mail: kezunov@ece.tamu.edu).

Color versions of one or more of the figures in this paper are available online at http://ieeexplore.ieee.org.

Digital Object Identifier 10.1109/TPWRD.2015.2464098 drops. Therefore, the control and analysis of PQ [3]-[6] provided to customers by the electricity utilities become even more important and necessary.

The main goal related to the operation of an electric power system is the continuous supply of energy, reliability, and minimal outages. Reference [7] provides best practices for monitoring $\mathrm{PQ}$, presenting a consistent description of electromagnetic phenomena typically observed in power systems.

In Brazil, there is a growing concern to standardize the quality control of electricity services. As a result, there is a government resolution [8] approving the procedures for operating electric power distribution systems, establishing the goals for the electric utilities with respect to the performance evaluation of their systems through measures of reliability, and setting the limit values to be accomplished and periodicity of performance assessments.

Faults occurring in distribution systems affect PQ in terms of service continuity and propagation of disturbances [9]. They are responsible for disturbances in industrial production processes, information loss, economic loss, and equipment breakdown, among others. An efficient fault location (FL) quickly and accurately helps minimize the time needed to find the problem and repair the system, speeding the power-supply re-establishment through the network restoration [10]. In addition, it helps reduce the costs of maintenance and operation. The system restoration can be performed with greater efficiency and speed when the fault location is known or, at least, can be estimated with good accuracy.

The fault-location program plays an important role in the short-term planning operation of electric distribution networks, reducing downtime and, hence. increasing the operational reliability of the distribution system. In the literature, a lot of works that propose different techniques to fault location on electric distribution systems [1], [11]-[20] can be found. In [11], [12], and [17], fault-location algorithms based on the phasors of fundamental frequency of voltages and currents measured at the feeder root node are proposed, as well as an apparent impedance computation. Iterative algorithms that compute the fault distance based on fundamental frequency phasors of voltages and currents measured at the feeder root node, as well as diagnosis and analysis of waveforms matching along with rejected loads after the fault clearing are proposed in [13]-[15]. In [18], the performances of 10 fault location methods for power distribution systems have been compared. The analyzed methods use only measurements of voltage and current at the substation. Fundamental components of prefault and during fault are used in these methods to estimate the apparent impedance viewed from 
the measurement point. Deviation between prefault and duringfault impedance, together with the system parameters, are used to estimate the distance to the fault point. Reference [20] proposes a fault-location technique that explores the voltage sag measurement and fundamentals of the short-circuit theory by using measurements from smart meters installed at the primary network.

The task of monitoring the PQ and fault detection, isolation, and network restoration (FDIR) may be very complicated in a DCC environment mainly in emergency conditions, where a large simultaneous flow of information from several monitored substations may overwhelm the operator. This paper focuses on an integrated approach in PQ and FL, through a reduced amount of measuring devices allocated in the distribution network, enabling the continuous analysis of a wide range of power system disturbances. The main contributions of this paper when compared to other approaches of the literature [1], [10], [17], [18] and [20] are:

- automatic detection of faults through analysis of oscillographic records of the three-phase currents, recorded at the substation;

- fast and accurate diagnosis of the fault type and PQ events through the combined use of the techniques, such as wavelet transform (WT), multiresolution analysis (MRA), and fuzzy-ARTMAP artificial neural network (ANN);

- geographic estimation of the faulty point based on analysis of electric circuits and voltage deviations, overcoming multiple estimation problems presented by most techniques;

- the integration of proposed algorithms in a modular design of the diagnostic system, which provides greater flexibility, precision, and high reliability for the obtained results.

Section II is devoted to intelligent algorithms for fault detection, feature extraction of behavioral indices, PQ analysis, short-circuit analysis, and fault location. The diagnosis of fault-type and PQ events present in the waveforms is accomplished through the combined use of wavelet-transform concepts [21], multiresolution analysis [22], and artificial neural networks (ANNs) [23]. The combination of such concepts in the design of the diagnostic system provides greater flexibility, accuracy, and high reliability of the obtained results. Section III provides tests results for all of the algorithms using a model of an actual distribution network in Brazil. Conclusions and references are given at the end.

\section{INTELLIGENT ALGORITHMS}

Regarding the detection, location, and classification of PQ disturbances, many computational tools have been proposed and used [16]-[20], however, none of them includes an integrated approach, seamlessly locating and analyzing the disturbances present in the electrical signals. The proposed approach focuses on several key issues related to PQ and fault location, such as dealing with limited information to locate faults, identifying sensitivities to errors, identifying and locating permanent and temporary faults, as well as experiencing performance improvements with the use of real data from distribution systems. Fig. 1

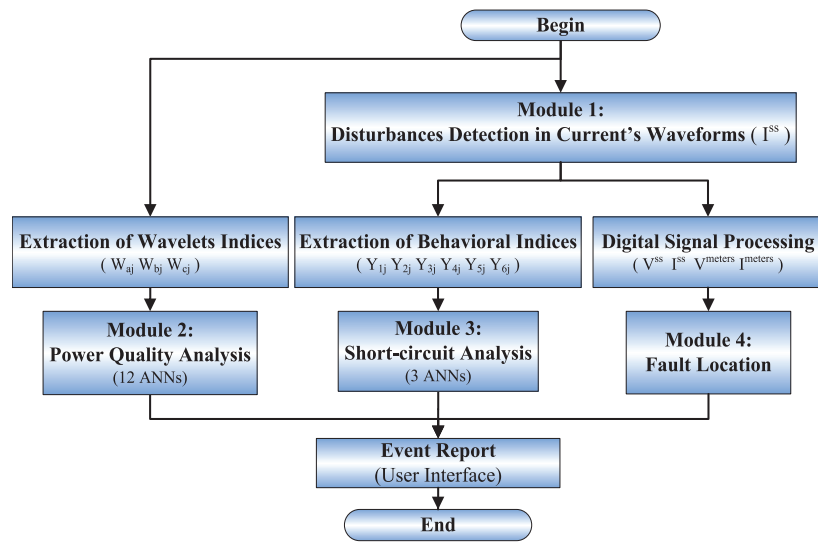

Fig. 1. Integrated intelligent algorithm for fault location and PQ analysis.

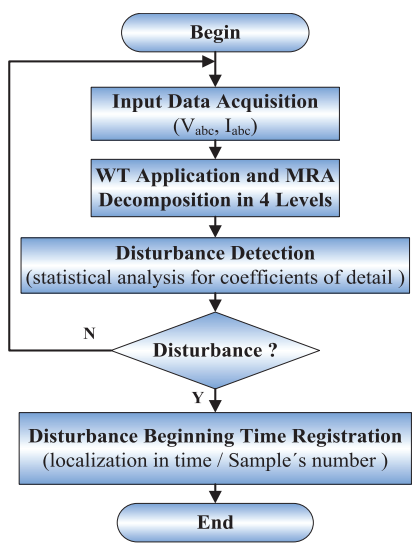

Fig. 2. Flowchart of the disturbances detection algorithm.

shows the flowchart of the proposed integrated intelligent algorithm for fault-location and $\mathrm{PQ}$ analysis. The main points related to the proposed methodology, regarding the four modules, are presented in the following subsections.

\section{A. Module 1: Fault Detection Algorithm}

The wavelet transform (WT) is a mathematical tool similar to the Fourier transform (FT) used for signal analysis, which can be used to extract important characteristics of different types of disturbances. WT is a modern signal-processing technique that overcomes the limitations of methods based on FT. Usually, the WT of transient signals is expressed by a multiresolution decomposition fast algorithm to decompose the signal to components under different scales. It is equal to successively filtering the signal with a high-pass and low-pass filter pair.

The fault detection algorithm is designed by taking into account the continuous monitoring of the current signal recorded from a measuring system present at the substation. This module analyzes the input signal using a sampling rate of $7.68 \mathrm{kHz}$, having a fixed window of 128 samples/cycle with a step of $1 / 4$ cycle. This data window is decomposed into MRA using the fourth-order Daubechies mother wavelet (db4). The analysis is performed for the first four levels of detail. Fig. 2 shows the flowchart of the disturbances detection algorithm. 

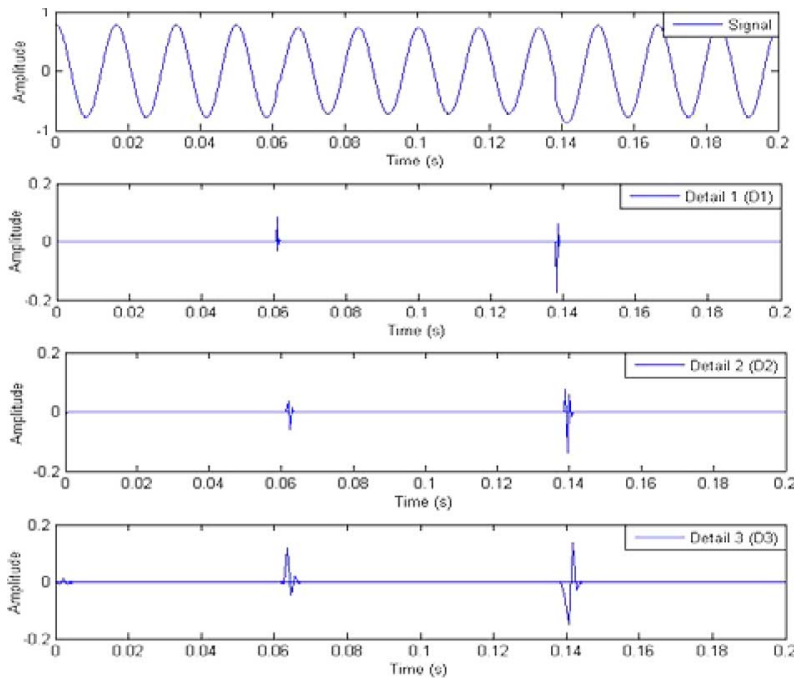

Fig. 3. MRA decomposition of a voltage sag.

The rules for the disturbances detection were created by observing the behavior of current signals in various situations of system operation. Thus, detection of a disturbance is based on the set of rules [10] presented as

$$
\text { if } \begin{aligned}
\max \left(\operatorname{var}_{i j}\right) & >3 \sigma_{i j} \text { and } \\
\max \left(\left|d_{i j}^{k}\right|\right) & >\lambda_{i j} \rightarrow \lambda_{i j}=1 \\
\text { if } \max \left(\operatorname{var}_{i j}\right) & \leq 3 \sigma_{i j} \text { or } \\
\max \left(\left|d_{i j}^{k}\right|\right) & \leq \lambda_{i j} \rightarrow \lambda_{i j}=0 .
\end{aligned}
$$

Through statistical analysis of variance and standard deviation of the sampled signal, the algorithm seeks to know the general behavior of the signal, comparing the maximum variance of the detail coefficients $\left(\operatorname{var}_{i j}\right)$ to a percentage of the standard deviation $\left(\sigma_{i j}\right)$, providing greater immunity to the presence of noise in the oscillographic records.

In order to analyze the maximum value of the detail coefficients $\left(\mathrm{d}_{i j}^{k}\right)$, punctual analysis is performed, facing the highest detail coefficient with a predetermined threshold value $\left(\gamma_{j}\right)$, defined, and based on the operating characteristics of each system. Fig. 3 shows a voltage sag, followed by the first (D1), second (D2), and third (D3) levels of detail, decomposed by MRA. It can be seen that the three first levels of detail correctly indicate the instants of beginning and ending of the disturbance through the illustrated peaks.

\section{B. Feature Extraction}

According to Parseval's theorem [24], the energy E of a discrete signal $x[n]$ can be calculated in both time domain and frequency domain as

$$
E_{i j}=\sum_{n=1}^{N_{j}}\left|x_{i}[n]\right|^{2}
$$

where $\mathrm{N}_{j}$ means the number of samples in the decomposition level $j, x_{i}[n]$ represents the nth sample coefficient of detail or approximation, and $i$ is the oscillographic record analyzed, that is, $\mathrm{I}_{a}, \mathrm{I}_{b}, \mathrm{I}_{c}, \mathrm{~V}_{a}, \mathrm{~V}_{b}$, or $\mathrm{V}_{c}$.
Since signals with PQ disturbances have large imbalances with different frequency components, the calculation of signal energy can be used to extract important characteristics of different types of disturbances. Thus, by calculating the energy of the signals prefault and postfault, it is possible to quantify the degree of imbalance in the operational status of the system. This must be a good tool to evaluate, discreetly, nonstationary signals. Fig. 4 presents the flowchart of the disturbances classification algorithm.

1) Representation of the Behavioral Indices: A relative representation between phases is employed for the representation of the behavioral indices [18]

$$
\begin{aligned}
Y_{1 j} & =X_{I_{a} j}-X_{I_{b} j}, Y_{2 j} \\
& =X_{I_{b} j}-X_{I_{c} j}, Y_{3 j}=X_{I_{c} j}-X_{I_{a} j} \\
Y_{4 j} & =X_{V_{a} j}-X_{V_{b} j}, Y_{5 j} \\
& =X_{V_{b} j}-X_{V_{c} j}, Y_{6 j}=X_{V_{c} j}-X_{V_{a} j}
\end{aligned}
$$

where $X_{i j}$ is the normalized value of the ratio between the postfault $E_{i j}^{d}$ and prefault $E_{i j}^{P}$ energies of the oscillographic record $i$ in the level of decomposition $j$. These representative indices lie between -1 and +1 . In order to be used as an input of the Fuzzy-ARTMAP neural networks, they need to be translated to the domain $\Omega=[0,+1]$

$$
Y_{n j}^{T}=\frac{Y_{n j}+1}{2}
$$

where $n$ represents the behavioral indices in analysis, that is, $\mathrm{n}=1,2,3,4,5$, or 6 . Thus, the Fuzzy-ARTMAP neural networks responsible for the short-circuit classification have as input some vectors composed by the behavioral indices $Y_{n j}^{T}$, which accurately characterize the phases involved in the disturbance.

2) Representation of the Voltage Wavelet Indices: The wavelet indices $W_{i j}$ are represented by the normalized and translated values of the difference between the prefault $E_{i j}^{P}$ and postfault $E_{i j}^{d}$ energies of the oscillographic record $i$ in the level of decomposition $j$

$$
W_{i j}=\frac{\frac{E_{i j}^{P}-E_{i j}^{d}}{\Delta_{V_{x} j}^{\max }}+1}{2}
$$

where $\Delta_{V_{x} j}^{\max }$ represents the maximum value related to the analysis of the voltage signals in the decomposition level $\mathrm{j}$, that is, $\Delta_{V_{x} j}^{\max }=\max \left\{\Delta_{V_{a} j}^{\max }, \Delta_{V_{b} j}^{\max }, \Delta_{V_{c} j}^{\max }\right\}$, and $x=a, b$, or $c$.

Thus, the Fuzzy-ARTMAP neural networks responsible for the classification of PQ events have as input some vectors composed by the wavelet indices, which satisfactorily characterize the PQ events.

\section{Module 2: $P Q$ Analysis}

The Fuzzy-ARTMAP neural networks are self-organizing networks that present supervised training, capable of identifying/classifying data in clusters. This architecture presents two key characteristics: 1) stability and 2) plasticity, which enable applications in modern electric power systems. These attributes enable continuous training to be incorporated while providing fast diagnosis and stable learning [23]. 


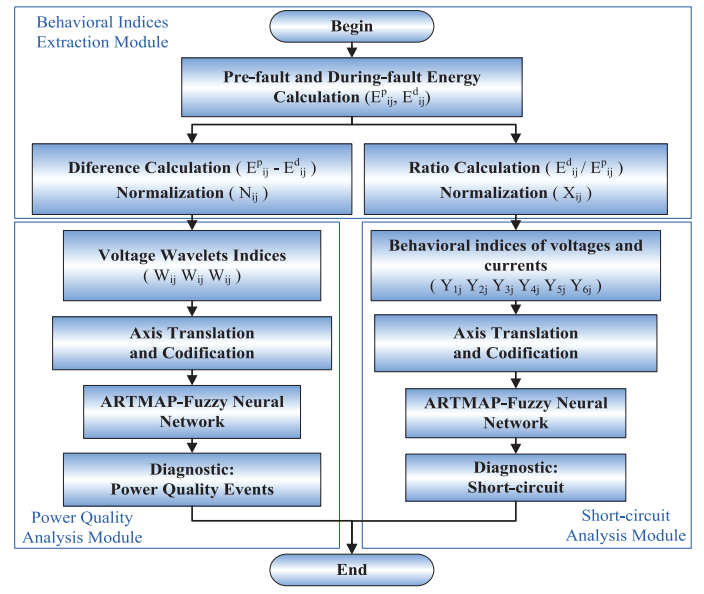

Fig. 4. Flowchart of the disturbances classification algorithm.

The PQ analysis module is responsible for the evaluation and classification of events recorded in the voltages waveforms. Its input vectors are the voltage wavelets indices calculated in the previous subsection. The six levels of detail and the first and sixth levels of approximations of the wavelets indices were used as input data to compose the input vector $\overline{I_{x}^{\text {in }}}$ for the ANNs. Thus, the input vector $\overline{I_{x}^{\text {in }}}$ has dimension 16 , which is composed of these eight wavelet indices approaches plus its complement

$$
\overline{I_{x}^{\text {in }}}=\left[\begin{array}{ll}
I_{x} & I_{x}^{c}
\end{array}\right]
$$

where

$$
\begin{aligned}
I_{x} & =\left[W_{V_{x} d_{1}} W_{V_{x} d_{2}} W_{V_{x} d_{3}} W_{V_{x} d_{4}} W_{V_{x} d_{5}} W_{V_{x} d_{6}} W_{V_{x} a_{1}} W_{V_{x} a_{6}}\right] \\
I_{x}^{c} & =\text { complement of } I_{x} .
\end{aligned}
$$

The output vector $\overline{I_{x}^{\text {out }}}$ used in the training phase of FuzzyARTMAP neural networks is encoded. This encoding of the input vector as well as the output vector is used for the three voltage oscillographic records recorded, that is, for the three phases of the system: $V_{a}, V_{b}$, and $V_{c}$.

\section{Module 3: Short-Circuit Analysis}

After determining the disturbance starting time, detection, and identification, the short-circuit diagnosis is carried out along with the PQ analysis. This is possible due to the modular structure employed to develop the detection and classification algorithms. The independency of the PQ analysis and short-circuit analysis modules is achieved by using distinct neural networks, independently trained.

Thus, three ANNs with different input patterns were created and trained. The first ANN has an input vector $\overline{I_{x}^{\text {in }}}$ with dimension 30 , consisting of the three first behavioral indices of the system with four levels of detail and the fourth level of approximation, plus its complement

$$
\begin{aligned}
I_{x}=\left[Y_{1 d_{1}}^{T} Y_{1 d_{2}}^{T} Y_{1 d_{3}}^{T} Y_{1 d_{4}}^{T} Y_{1 a_{4}}^{T} Y_{2 d_{1}}^{T} Y_{2 d_{2}}^{T} Y_{2 d_{3}}^{T} Y_{2 d_{4}}^{T}\right. \\
\left.Y_{2 a_{4}}^{T} Y_{3 d_{1}}^{T} Y_{3 d_{2}}^{T} Y_{3 d_{3}}^{T} Y_{3 d_{4}}^{T} Y_{3 a_{4}}^{T}\right]
\end{aligned}
$$

The second ANN is designed using an input vector $\overline{I_{x}^{\text {in }}}$ also with dimension 30 , composed by the last three behavioral indices,

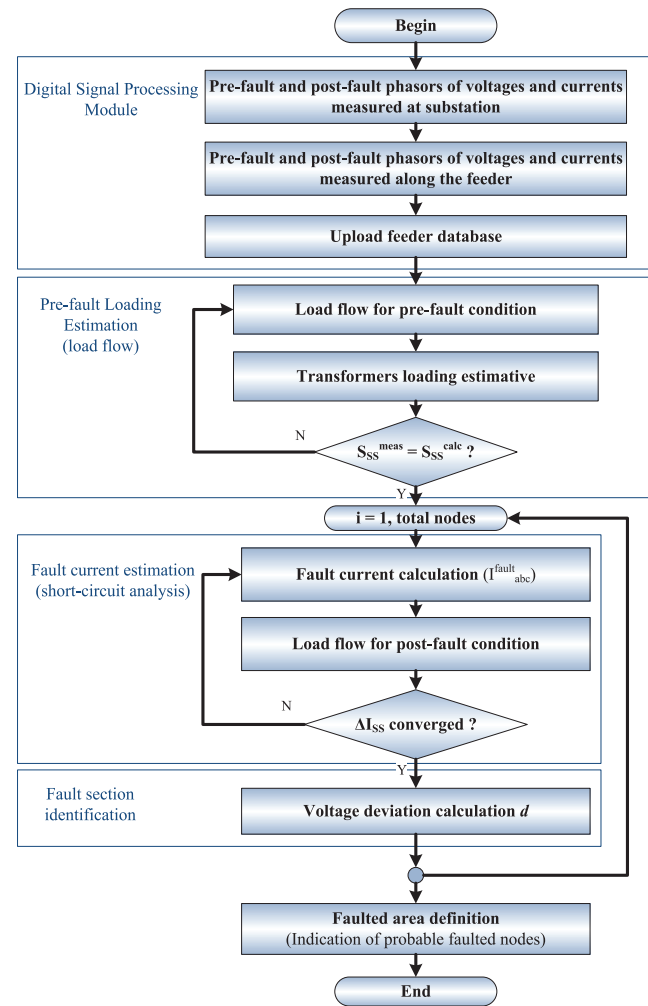

Fig. 5. Flowchart of the FL algorithm.

obtained from oscillographic records of voltages recorded at the substation

$$
\begin{array}{r}
I_{x}=\left[Y_{4 d_{1}}^{T} Y_{4 d_{2}}^{T} Y_{4 d_{3}}^{T} Y_{4 d_{4}}^{T} Y_{4 a_{4}}^{T} Y_{5 d_{1}}^{T} Y_{5 d_{2}}^{T} Y_{5 d_{3}}^{T} Y_{5 d_{4}}^{T}\right. \\
\left.Y_{5 a_{4}}^{T} Y_{6 d_{1}}^{T} Y_{6 d_{2}}^{T} Y_{6 d_{3}}^{T} Y_{6 d_{4}}^{T} Y_{6 a_{4}}^{T}\right]
\end{array}
$$

The third ANN was designed and trained using an input vector $\overline{I_{x}^{\text {in }}}$ with dimension 60 , composed of by the six behavioral indices of the system, referring to the current and voltage oscillographic records recorded at the substation.

After the execution of the detection algorithm and the classification of disturbances, which provides an overview of the operational status of the network, the process of geographical fault location begine, through the FL algorithm.

\section{E. Module 4: Fault Location}

The FL procedure consists of automatically determining the geographical location of a fault by performing digital signal processing of the waveforms of voltages and currents. They are measured at the substation level and a minimum set of points placed along the feeder in order to calculate the voltage deviations. These waveforms are obtained through the use of smart meters - protective and control equipment with intelligent sensors, such as automatic switchgear, reclosers or devices equipped with remote terminal units (RTUs).

Fig. 5 shows the flowchart of the FL algorithm developed based on the algorithms of backward/forward sweep power flow [25] and short-circuits analysis [26], which is proven to be efficient and appropriate for radial or weakly meshed distribution networks, even in the presence of distributed generation. 
Besides the measurement data, the algorithm needs a database containing information about the network topology, that is, impedances of the lines sections and the power rating of distribution transformers. Reference [17] explains in a more detailed way the FL methodology. The main aspects of the FL algorithm are presented in the following subsection.

1) Data Acquisition and Digital Signal Processing: The digital signal processing of the currents and voltages in order to obtain the prefault and postfault phasors of voltages and currents, necessary for performing the FL algorithm, is carried out through the fast Fourier transform (FFT). The sampling of the current signals is also used to identify the fault type by means of a Fuzzy-ARTMAP neural network that identifies the type and the phases involved in the fault. Dedicated devices installed along the feeder should make remote measurements of prefault and postfault voltages available. Communication channels should also be available for the transmission of the measured data points to the remote processing site of the FL algorithm.

2) Prefault Loading Estimation: The prefault power output of the feeder is calculated using phasors of phase currents and voltages. These phasors are used to calculate the prefault apparent power supplied by the feeder, which is used to estimate the distribution transformers load before the instance of the fault occurrence. The load of each distribution transformer is estimated based on its nominal power. Therefore, prefault complex power of each transformer can be calculated according to the following equation:

$$
S_{i}=\rho_{i} S_{i}^{\text {nom }} \frac{S_{S S}}{\sum_{k=1}^{n t} \rho_{k} \cdot S_{k}^{\text {nom }}}\left[\cos \left(\varphi_{S S}\right)+j \sin \left(\varphi_{S S}\right)\right]
$$

where $S_{i}^{\text {nom }}$ is the rated power of the $i$ th transformer $S_{S S}$ is the apparent power estimated at the substation level, $\cos \left(\varphi_{S S}\right)$ is the substation power factor, $n t$ is the total number of transformers, and $\rho_{i}$ is the mean loading of the $i$ th transformer.

3) Fault Current Estimation: Fault current is estimated considering that voltages and currents at all nodes in the system (prefault power flow) and the postfault current at SE are known. A fault in the distribution system is treated as a load connected to the feeder. The fault current $I_{f}$ is calculated using (12) at each iteration and then is injected into the analyzed node

$$
I_{f}=I_{S S}-\sum_{i=1}^{n t} I_{i}
$$

where $I_{s s}$ is the postfault current measured at substation and $I_{i}$ is the loading current of the ith distribution transformer.

The use of these current injections does not require any assumption regarding the fault impedance, since it does not play a role in the fault modeling.

4) Faulty Section Estimation: Faults generally produce voltage sags with different magnitudes; however, in some cases, voltage sags of the same magnitude can be found. While voltage sags can be equal, their respective phase angles are not. Since faults are affected mainly by the resistive part of the fault impedance, they will produce different changes in phase angles compared to line impedances. Thus, the multiple estimation

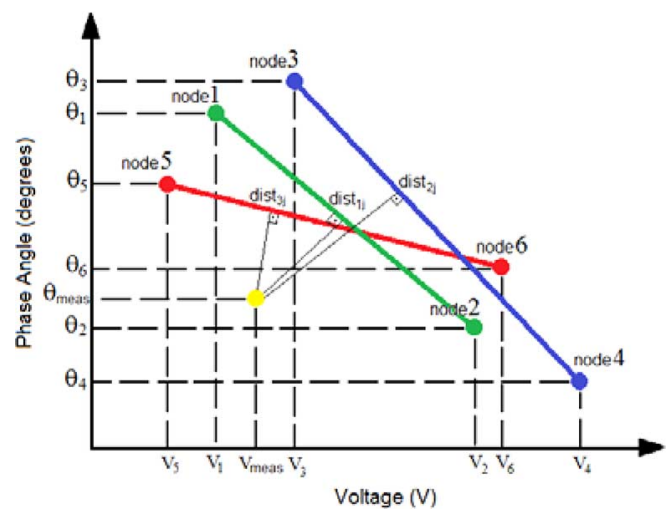

Fig. 6. Distance between the faulty point and the candidate lines.

problem presented by most of the techniques is overcome by using the change in phase angle along with the magnitude of the voltage sag.

Defining and identifying the faulty section requires the computation of the voltage deviations $\left(\right.$ delta $\left._{i}\right)$ through the indication of likely faulty nodes, using the distances $\left(d i s t_{i j}\right)$, calculated for each line $i$ of the system with $j$ measuring devices

$$
\operatorname{dist}_{i j}=\frac{\left|a V_{i}^{\text {meas }}-\theta_{i}^{\text {meas }}+c\right|}{\sqrt{a^{2}+1}}
$$

where $d_{i s t_{i j}}$ is the distance between the measured and calculated faulty point for the bus $i$, referring to the meter $j, V_{i}^{\text {meas }}$ is the voltage measured, $\theta_{i}^{\text {meas }}$ is the phase angle measured, $a$ is the angular coefficient, and $c$ is the linear coefficient. So the voltage deviations are calculated using the maximum and minimum distances for each $\mathrm{k}$ phase.

$$
\operatorname{delta}_{i}=\max \left\{\frac{1}{\max \left\{d i s t_{i j}^{k}\right\}-\min \left\{d i s t_{i j}^{k}\right\}+\varepsilon}\right\}
$$

where $\varepsilon$ is a small number to avoid division by zero, and $\mathrm{k}$ is the phase $a, b$, or $c$.

Fig. 6 shows an example of the distance calculation $\left(\right.$ dist $\left._{i j}\right)$ between the faulty point and the candidate branches/lines. Thus, the line that connects busbars 5 and 6 (red line) has the lowest distance $\left(\right.$ dist $\left._{3 j}\right)$ in relation to the measured value (yellow point). Therefore, it is possible to sort the lines according to the distance error found for each measuring device.

By sorting the results of (14) in increasing order and normalizing the maximum value in relation to $\operatorname{delta}_{i}^{\mathrm{MAX}}$ found in all samples, there is the final ranking of the fault location

$$
f l_{i}=\left(\frac{\operatorname{delta}_{i}}{\operatorname{delta}_{i}^{\mathrm{MAX}}}\right) * 100 .
$$

Thus, (15) gives the probable classification nodes or branches on a scale ranging from 0 to $100 \%$, with the highest value of $f l_{i}$ corresponding to the biggest variation in $\operatorname{delta}_{i}$.

\section{CASE Study}

A real-life three-phase distribution system with 134 nodes-13.8 kV and 7.065 MVA (Fig. 7) was used to test the proposed algorithms [27]. The fault location is performed after the fault detection and classification process. The proposed 


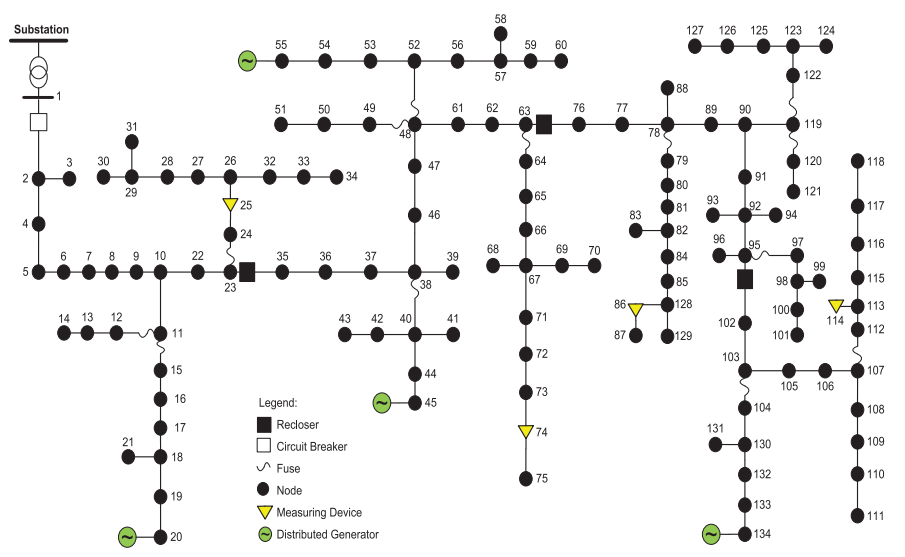

Fig. 7. Real-life three-phase distribution system [27].

TABLE I

Detection of a Current Disturbance in Phase A

\begin{tabular}{ccccc}
\hline Window & $\max \left[\operatorname{var}_{i j}\right]$ & $3 \sigma_{i j}$ & $\max \left[\left|d_{i j}^{k}\right|\right]$ & $\gamma_{j}$ \\
\hline$\vdots$ & $\vdots$ & $\vdots$ & $\vdots$ & $\vdots$ \\
15 & 227,5 & 326,7 & 314,0 & 281,2 \\
16 & 174,5 & 296,8 & 297,8 & 281,6 \\
17 & 227,6 & 326,7 & 314,3 & 281,2 \\
$\mathbf{1 8}$ & $\mathbf{8 2 3 , 7}$ & $\mathbf{5 7 1 , 3}$ & $\mathbf{5 4 4 , 6}$ & $\mathbf{2 8 1 , 6}$ \\
\hline
\end{tabular}

technique applies to three-phase radial or weakly meshed feeders with unbalanced loads, lateral extensions with one, two, or three phases and presents distributed generation.

\section{A. Fault Detection Algorithm}

The disturbance detection is based on the set of rules presented in (1) and (2), which are composed by a) macroanalysis and b) microanalysis. Through a), the signal general behavior is examined, comparing the maximum variation of the detail coefficients with a standard deviation percentage, which provides major immunity to the noise presence in oscillographies. The threshold plays an important role for the sensitivity to a fault because it performs punctual analysis in $b$ ), analyzing the maximum value of the detail coefficients $\left(\mathrm{d}_{i j}^{k}\right)$ and facing the highest detail coefficient with a predetermined threshold value $\left(\gamma_{j}\right)$. The thresholds are defined by the protection engineer, based on the operating characteristics of each system.

The evolution of a signal containing a short circuit caused by a phase-to-ground fault of $20.0 \Omega$ at node 43 , starting at 0.08333 $\mathrm{s}$ is considered to illustrate the detection of a disturbance and its current location time. Table I presents the signal detection window and its respective statistical analysis of variance and standard deviation.

In this situation, the fault was detected after $0.08372 \mathrm{~s}$ related to the sample number 323 of the recorded signal. This result was found during the analysis of the 18th window sampled and decomposed by MRA, considering the set of rules presented in (1) and (2). This module reached $100 \%$ accuracy with highcomputing performance.
Following the detection of the disturbance in a sampled signal, the algorithm records 10 complete cycles of this signal, with four prefault cycles and six postfault cycles and, then, starts the PQ analysis stage.

\section{B. $P Q$ Analysis}

The PQ events were generated by summing the steady-state sinusoidal waveform, which generated at a sampling rate of 7.68 $\mathrm{kHz}$ through Alternative Transients Program/Electromagnetic Transients Program (ATP/EMTP) software [28], with a vector containing the respective disorder. The disturbance amplitude is estimated using the peak values for the voltage level of the first approach (cA1), before and during the disturbance. The difference between such values indicates the variation in signal amplitude that characterizes the disturbance.

Fig. 8 shows the first version of approximation (cAla) of a signal containing a voltage sag in phase A of node 25 . In this example, the peak value before the disturbance has a value equal to 10379.07 , and the value during the disturbance is equal to 8584.06 , corresponding to a variation in the disturbance amplitude of $17.3 \%$.

Thereafter, 12 ANNs were trained and used to classify the PQ events. Thus, each measuring device needs three ANNs, one for each phase, and the bigger the number of measurement devices, the larger the amount of ANN required. Each ANN was trained using a set of 11704 simulations and then tested with the simulations reported in the other measuring devices placed along the network. The vigilance parameters $\rho_{a}$ and $\rho_{b}$ adopted for the training of the 12 neural networks were 0.85 and 0.95 , respectively. The inter-ART parameter used was 0.99 and the training rate for both networks $\mathrm{ART}_{a}$ and $\mathrm{ART}_{b}$ was 1.0.

Table II shows the operational efficiency of ANNs trained to classify PQ events occurring in phase A. The input patterns of these networks are composed by energy coefficients of the first four levels of details and approximations of current and voltage signals, and the output network presents the fault-type code and the phases involved in the problem.

The events most frequently reported by ANNs after evaluation of all networks are the ones that will prevail, and these events will appear in the output report. The high performance of these ANNs in the classification process of PQ disturbances can be clearly verified.

\section{Short-Circuit Analysis}

The training and testing of the three ANNs responsible for the short-circuit classification were carried out using the waveforms of voltages and currents simulated using ATP software, and applying fault situations in various parts of the system, inserting these faults with various incidence angles and different values of fault resistances. Table III shows the variables used in short-circuit simulations.

After training these three ANNs with the 17556 fault simulations previously performed, in order to verify the efficiency of the classification process, another 11704 simulations were performed using the variables shown in Table III, but now considering the angles of incidence of failure as being 30 and $60^{\circ}$. 


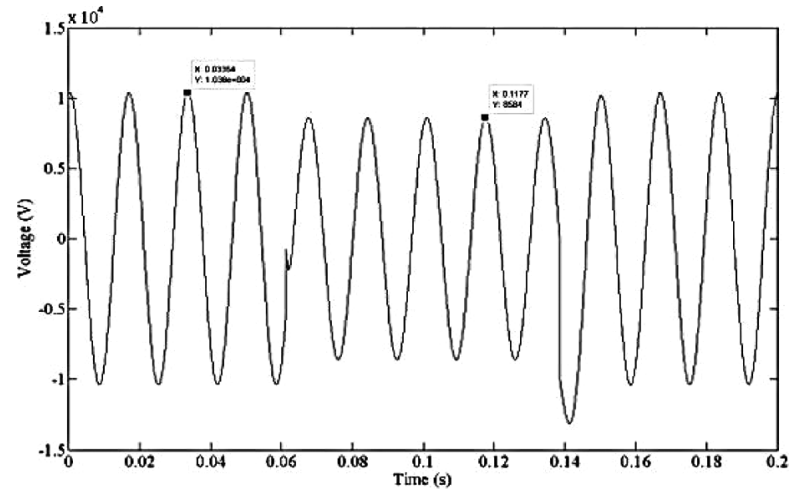

Fig. 8. Waveform of a voltage sag in phase A of node 25 .

TABLE II

OPerational EFficiency of ANNs Trained for PQ Events in Phase A

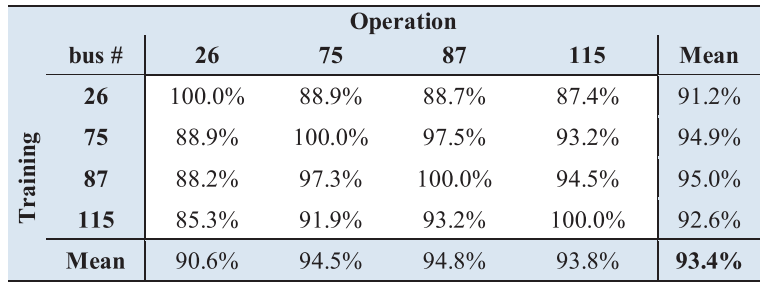

TABLE III

VARIABLES USED IN SHORT-CIRCUIT SIMULATIONS

\begin{tabular}{|c|c|c|}
\hline Variable & Variation & Total \\
\hline Fault resistance $(\Omega)$ & {$\left[\begin{array}{llll}10 & 20 & 30 & 40\end{array}\right]$} & 4 \\
\hline Incidence angle & [0 45 90] & 3 \\
\hline Fault type & $\begin{array}{c}\text { A B C AB ABG BC BCG } \\
\text { CA CAG ABC ABCG ] }\end{array}$ & 11 \\
\hline \multirow[t]{2}{*}{ Faulted bus } & {$\left[\begin{array}{llllllll}2 & 3 & 4 & 5 & 6 & 7 & \ldots & 134\end{array}\right]$} & 133 \\
\hline & Total & 17,556 \\
\hline
\end{tabular}

TABLE IV

ANNS EFFICIENCY FOR SHORT-CIRCUIT ANALYSIS

\begin{tabular}{c|c|c}
\hline ANN & Behavioral Indices & Efficiency \\
\hline 1 & $Y_{1 j}^{T}, Y_{2 j}^{T}$ and $Y_{3 j}^{T}$ & $97.3 \%$ \\
\hline 2 & $Y_{4 j}^{T}, Y_{5 j}^{T}$ and $Y_{6 j}^{T}$ & $99.2 \%$ \\
\hline 3 & $Y_{1 j}^{T}, Y_{2 j}^{T}, Y_{3 j}^{T}, Y_{4 j}^{T}, Y_{5 j}^{T}$ and $Y_{6 j}^{T}$ & $99.8 \%$ \\
\hline
\end{tabular}

Table IV shows the efficiency of the classification process encountered during the tests with the three ANNs.

The high performance achieved by the three ANNs can be clearly observed. The error for three-phase faults in relation to the presence of ground or not was pretty much the same.

\section{Fault Location}

The fault-location algorithm proposed to analyze the operational state of the distribution systems requires the oscillographic records of the currents and voltages from the measuring system present at the substation (feeder root node) and the voltages measured at some remote points, located along the feeder. A sequence of random variables indexed in time with a well-defined correlation structure was considered to simulate the operating conditions of a real system as a stochastic process. Table $\mathrm{V}$
TABLE V

Stochastic Variables Used in SimUlations

\begin{tabular}{c|c|c|c|c}
\hline Variable & Distribution & Mean & $\begin{array}{c}\text { Std. } \\
\text { Deviation }\end{array}$ & Variation \\
\hline Faulted Bus & Uniform & - & - & 2 to 134 \\
\hline Fault Phase & Uniform & - & - & A, B ou C \\
\hline Fault type & Normal & 0 & 1 & $\begin{array}{c}5 \% \text { three-phase } \\
10 \% \text { line-to-line } \\
85 \% \text { single-line-to-ground }\end{array}$ \\
\hline $\begin{array}{c}\text { Line-to-ground } \\
\text { resistance }\end{array}$ & Normal & 25 & 4.854 & $10-40$ \\
\hline $\begin{array}{c}\text { Line-to-line } \\
\text { resistance }\end{array}$ & Normal & 0.5 & 0.129 & $0.1-0.9$ \\
\hline Loading & Uniform & - & - & high, medium, low \\
\hline
\end{tabular}

TABLE VI

Mean Values for Stochastic Simulations Results

\begin{tabular}{c|c|c|c|c|c}
\hline \multirow{2}{*}{ DG } & Loading & $\begin{array}{c}\text { Line-to-line } \\
\text { resistance }\end{array}$ & $\begin{array}{c}\text { Line-to-ground } \\
\text { resistance }\end{array}$ & Position & $\begin{array}{c}\text { Probability } \\
(\mathbf{\%})\end{array}$ \\
\hline \multirow{4}{*}{ No } & High & 0.5 & 25.1 & 5 & 89.7 \\
\cline { 2 - 6 } & Medium & 0.5 & 25.2 & 6 & 89.6 \\
\cline { 2 - 6 } & Low & 0.5 & 25.0 & 5 & 91.0 \\
\hline \multirow{3}{*}{ Yes } & High & 0.5 & 24.9 & 8 & 79.5 \\
\cline { 2 - 6 } & Medium & 0.5 & 25.2 & 9 & 78.2 \\
\cline { 2 - 6 } & Low & 0.5 & 25.0 & 9 & 79.6 \\
\hline
\end{tabular}

shows the random variables considered in the simulations, with their respective variation ranges.

Five-thousand faults were simulated for the distribution system with and without the presence of DG. Table VI shows the average values of the results obtained from the stochastic simulations, after 5000 simulations. The precision of the FL algorithm must be verified by the small mean position found in Table VI and the high mean probability presented by the stochastic simulations.

The average time taken to locate the faulty points is less than $30 \mathrm{~s}$ in all cases. It can be considered to be a good computational time since the repairing time and dispatching of maintenance crews take much longer. Fig. 9 shows the confidence intervals of the probabilities of faults occurrence and the positions classified by the FL algorithm, for each fault type simulated. The presence of distributed generation is considered in the calculation of power flow and short-circuit analysis, and the fault resistance can be estimated at the end of the process by the currents and voltages encountered after their convergence, since the faults occurring in the system are modeled as current injections.

For each bus, a feeder was applied with a line-to-ground fault occurring in phase A, considering a line-to-ground resistance of $10 \Omega$ and medium loading modeled like constant impedance. Fig. 10 shows the classification of buses for a fault occurring in bus 70 . The FL system had no problem classifying the fault because the voltage deviations showed quite different values, which shows good accuracy of the FL system.

Fig. 11 shows the sum of the actual positions of the buses located on the classification made by the FL algorithm for a line-to-ground fault occurring in phase A.

The number and position of measuring devices affect the performance of the FL program. However, the number of measuring devices that must be allocated on the network is minimal, different from [20], which uses a greater number of measuring devices, increasing the data traffic and computational effort, and 

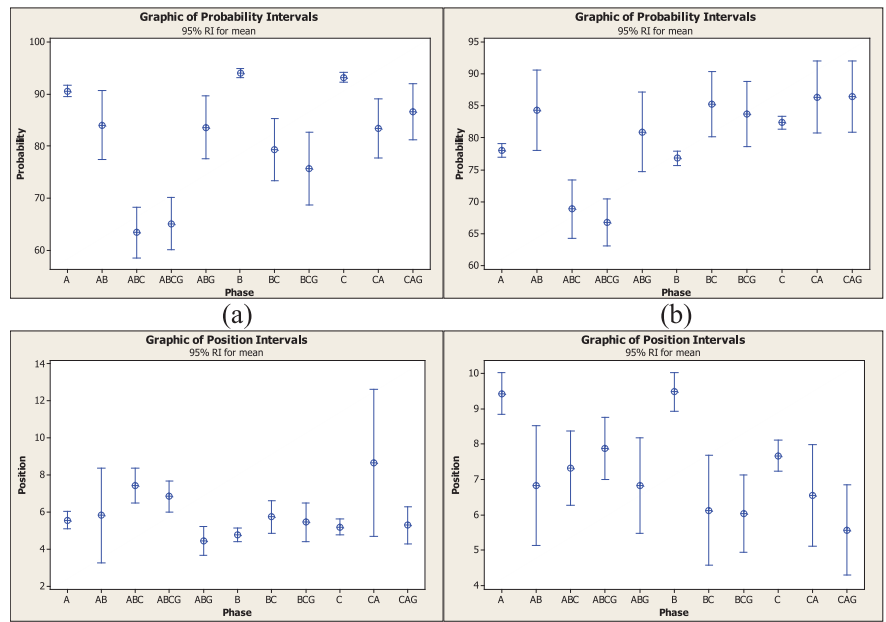

(c)

(d)

Fig. 9. Confidence intervals of the probabilities of faults occurrence and the positions classified by the FL algorithm. (a) Probability without DGs. (b) Probability with DGs. (c) Position without DGs. (d) Position with DGs.

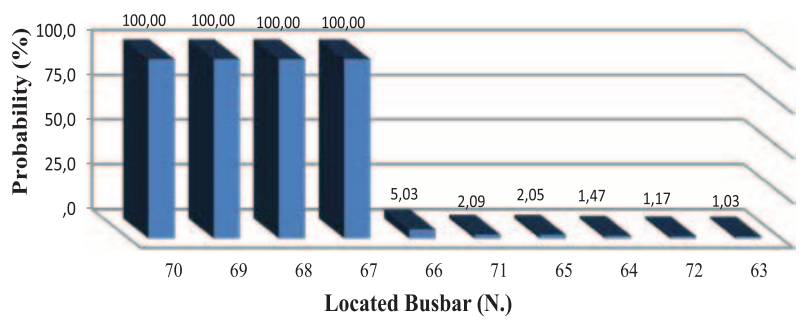

Fig. 10. Classification of buses for a fault occurring in busbar 70 .

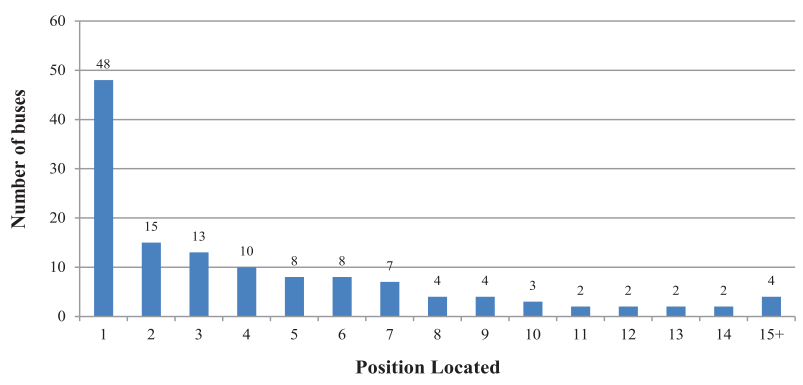

Fig. 11. Number of buses classified by position.

whose methodology only works for distribution systems with a high level of automation.

The efficiency and versatility of the developed intelligent algorithms to locate faults and monitor PQ can be clearly observed. When used together, as shown here, they provide a great diagnosis of the operating conditions of the distribution network, either operating normally or under contingencies.

\section{CONCLUSIONS}

A methodology for integrated, automated diagnosis, and FL through the combined usage of modern techniques of network analysis, signal processing, and intelligent systems was presented in this paper. The continuous (online) process of fault detection is accomplished through statistical analysis and direct current multilevel signal analysis in the wavelet domain, recorded at the substation. The sampling rate adopted in this work was $7.68 \mathrm{kHz}$, representing 128 samples/cycle. This is not a very high frequency and can be easily applied. The disturbance detection process is accomplished using the fourth-order Daubechies (db4) wavelet filter in MRA. The behavioral indices of the current and voltage signals are extracted by employing the DWT, multiresolution analysis, and the concept of signal energy. These proposed indices correspond to the input vectors of a number of independent Fuzzy-ARTMAP NNs, which aim to estimate the fault-type and PQ events present in the sampled signal.

The FL is performed after the classification process, and it is applicable to three-phase radial or weakly meshed feeders with unbalanced loads, three phases, and double-line or, single-line lateral extensions and it works with the presence of DG.

The integrated system diagnosis and FL are able to assist the electric utility personnel in their daily job in providing important information for operation and maintenance, minimizing the repair time, and, consequently, experiencing lesser financial losses due to the ability for rapid fault diagnosis and restoration to normal system operation.

The proposed methodology can be applied in online operation to identify PQ events and is still valid even after network reconfiguration since the Fuzzy-ARTMAP ANNs are able to perform pattern recognition, independent of network configuration.

\section{REFERENCES}

[1] M. Kezunovic, "Smart fault location for smart grids," IEEE Trans. Smart Grid, vol. 2, no. 1, pp. 11-22, Mar. 2011.

[2] M. H. J. Bollen, Understanding Power Quality Problems: Voltage Sags and Interruptions. New York, USA: Wiley/IEEE, 2000, p. 543.

[3] M. H. J. Bollen and I. Y. H. Gu, Signal Processing of Power Quality Disturbances. Hoboken, NJ, USA: Wiley/IEEE, 2006, p. 861.

[4] M. H. J. Bollen, "Voltage sag: Effects, mitigation and prediction," Power Eng. J., vol. 10, no. 3, pp. 129-135, Jun. 1996.

[5] G. Olguin, F. Vuinovich, and M. H. J. Bollen, "An optimal monitoring program for obtaining Voltage sag system indexes," IEEE Trans. Power Syst., vol. 21, no. 1, pp. 378-384, Feb. 2006.

[6] IEEE Guide for Identifying and Improving Voltage Quality in Power Systems, IEEE Standard 1250-2011, Mar. 2011.

[7] IEEE Recommended Practice for Monitoring Electric Power Quality, IEEE Standard 11590-2009, Jun. 2009.

[8] Procedures for Electric Power Distribution in the National Electric System, Normative Resolution N. 395, Dec. 2009.

[9] I. D. Suarez, G. C. Caicedo, and H. R. Vargas-Torres, "Review of stable and transient fault location techniques for distribution systems Part II: Steady-state knowledge based and transient analysis techniques," in Proc. Int. Conf. Renew. Energies Power Qual., 2010, pp. 1-6.

[10] J. G. M. S. Decanini, M. S. Tonelli-Neto, and C. R. Minussi, "Robust fault diagnosis in power distribution systems based on Fuzzy ARTMAP neural network-aided evidence theory," IET Gen., Transm. Distrib., vol. 6, no. 11, pp. 1112-1120, Jul. 2012.

[11] A. A. Girgis, C. M. Fallon, and D. L. Lubkeman, "A fault location technique for rural distribution feeders," IEEE Trans. Ind. Appl., vol. 29, no. 6, pp. 1170-1175, Nov. 1993.

[12] R. Das, M. S. Sachdev, and T. S. Sidhu, "A fault locator for radial subtransmission and distribution lines," in Proc. IEEE Power Eng. Soc. Summer Meeting, 2000, pp. 443-448.

[13] J. Zhu, D. L. Lubkeman, and A. A. Girgis, "Automated fault location and diagnosis on electric power distribution feeders," IEEE Trans. Power Del., vol. 12, no. 2, pp. 801-809, Apr. 1997.

[14] S. J. Lee, M. S. Choi, S. H. Kang, B. G. Jin, D. S. Lee, B. S. Ahn, N. S. Yoon, H. Y. Kim, and S. B. Wee, "An intelligent and efficient fault location and diagnosis scheme for radial distribution systems," IEEE Trans. Power Del., vol. 19, no. 2, pp. 524-532, Apr. 2004.

[15] E. C. Senger, G. Manassero, C. Goldemberg, and E. L. Pellini, "Automated fault location system for primary distribution networks," IEEE Trans. Power Del., vol. 20, no. 2, pt. 2, pp. 1332-1340, Apr. 2005. 
[16] H. R. Diaz and M. T. Lopez, "Fault location techniques for electrical distribution networks: A literature survey," in Proc. Int. Power Energy Syst. Conf., 2005, pp. 311-318.

[17] R. A. F. Pereira, M. Kezunovic, and J. R. S. Mantovani, "Improved fault location on distribution feeders based on matching during-fault voltage sags," IEEE Trans. Power Del., vol. 42, no. 2, pp. 852-862, Apr. 2009.

[18] J. Mora-Florez, J. Melendez, and G. Carrillo-Caicedo, "Comparison of impedance based fault location methods for power distribution systems," Elect. Power Syst. Res., vol. 78, no. 4, pp. 657-666, Apr. 2008

[19] I. D. Suarez, G. C. Caicedo, and H. R. Vargas-Torres, "Review of stable and transient fault location techniques for distribution systems Part I: Steady-state locators based on circuit analysis," in Proc. Int. Conf. Renew. Energies Power Qual., 2010, pp. 1-8.

[20] F. C. L. Trindade, W. Freitas, and J. C. M. Vieira, "Fault location in distribution systems based on smart feeder meters," IEEE Trans. Power Del., vol. 29, no. 1, pp. 251-260, Feb. 2014.

[21] C. H. Kim and R. Aggarwal, "Wavelet transforms in power systems Part I - General introduction to the wavelet transforms," Power Eng. $J$., vol. 14, no. 2, pp. 81-87, Apr. 2000.

[22] C. S. Burrus, R. A. Gopinath, and H. Guo, Introduction to Wavelets and Wavelet Transforms: A Primer. Upper Saddle River, NJ, USA: Prentice-Hall, 1998, p. 268.

[23] G. A. Carpenter, S. Grossberg, N. Markuzon, J. H. Reynolds, and D. B. Rosen, "Fuzzy ARTMAP: A neural network architecture for incremental supervised learning of analog multidimensional maps," IEEE Trans. Neural Netw., vol. 3, no. 5, pp. 698-713, Sep. 1992.

[24] A. V. Oppenheim, R. W. Schafer, and J. R. Buck, Discrete-Time Signal Processing. Upper Saddle River, NJ, USA: Prentice-Hall, 1998, p. 870.

[25] C. S. Cheng and D. Shirmohammadi, "A three-phase power flow method for real-time distribution system analysis," IEEE Trans. Power Syst., vol. 10, no. 2, pp. 671-679, May 1995.

[26] X. F. Zhang, F. Soudi, D. Shirmohammadi, and C. S. Cheng, "A distribution short circuit analysis approach using hybrid compensation method," IEEE Trans. Power Syst., vol. 10, no. 4, pp. 2053-2059, Nov. 1995.

[27] "Power system test case archive," Sao Paulo, Brazil, 2014. [Online] Available: http://www.feis.unesp.br/Home/departamentos/engenhariaeletrica/lapsee/system_135_3ph_distribution_feeder.rar

[28] "ATP Rulebook. Revista Iberoamericana del ATP," Comite Argentino de Usuarios del EMTP/ATP, Dec. 2002. [Online]. Available: https://sites.google.com/site/atpcaue/reviberoamericana/ anio 2001/vol3 num4

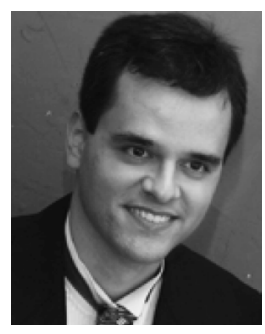

A. A. P. Bíscaro (M’09) received the Dipl.Ing., M.S., and Ph.D. degrees in electrical engineering from Universidad Estadual Paulista, Sao Paulo, Brazil, in 2005, 2009, and 2013, respectively.

Currently, he is a Full Professor of Electrical Engineering and a member of Grupo de Pesquisa em Energia, Eletrônica e telecomunicações at Universidad do Estado de Mato Grosso, Mato Grosso, Brazil. His main research areas are optimization methods, reliability, fault location, and protection of electric power systems.

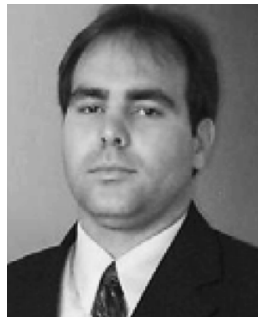

R. A. F. Pereira received the B.Sc., M.S., and Ph.D. degrees in electrical engineering from UNESP/Ilha Solteira, São Paulo, Brazil, in 2001, 2003, and 2007, respectively.

His research areas are fault location on distribution feeders as well protection and reliability of electric distribution systems.

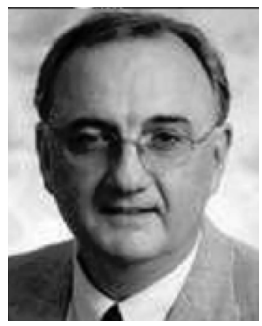

M. Kezunovic (S'77-M'80-SM'85-F'13) received the Dipl.Ing. degree in electrical engineering from the University of Sarajevo, Sarajevo, Yugoslavia, in 1974, and the M.S. and Ph.D. degrees in electrical engineering from the University of Kansas, Lawrence, in 1977 and 1980, respectively.

He has been with Texas A\&M University, College Station, TX, USA, since 1987, where he is the Eugene E. Webb Professor and Director of Electric Power and Power Electronics Institute. He was previously with Westinghouse Electric Corporation, Pittsburgh, PA, USA, and the Energoinvest Company, Sarajevo, and was a Visiting Associate Professor at Washington State University during 1986-1987. His main research interests are digital simulators and simulation methods for equipment evaluation and testing, as well as application of intelligent methods to control, protection, and power-quality monitoring.

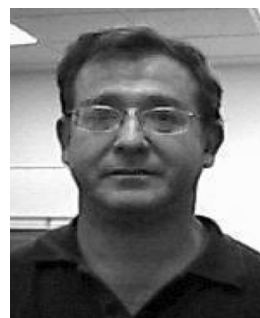

J. R. S. Mantovani (M’03) received the Dipl.Ing. degree in electrical engineering from Univ Estadual Paulista, Sao Paulo, Brazil, in 1981 and the M.S. and $\mathrm{Ph} . \mathrm{D}$. degrees from UNICAMP - Univ de Campinas, Sao Paulo, Brazil, in 1987 and 1995, respectively.

Currently, he is a Full Professor of Electrical Engineering and a member of Laboratório de Planejamento de Sistemas de Energia Elétrica in the Department of Electrical Engineering of Universidad Estadual Paulista, Sao Paulo, Brazil. His main research interests are the planning and control of electric power systems. 